\title{
Über die «Kohlenhydratgruppe» in der echten Nucleinsäure. \\ II. Mitteilung.
}

\author{
Von \\ R. Fealgen. \\ (Aus dem physiologischen Institut der Universität Berlin.) \\ (Der Redaktion zugegangen am 1. August 1917.)
}

Das Molekulargewicht der echten Nucleinsäure steht bis jetzt noch nicht mit Sicherheit fest, und es gibt deswegen nicht einmal eine allgemein anerkannte empirische Formel.

Man kann die Arbeiten, die auf die Aufstellung einer Formel für die echte Nucleinsäure hinzielen, in drei Perioden einteilen, die auch zeitlich aufeinander folgen.

In der ersten Periode war die Zusammensetzung der Nucleinsäure hinsichtlich ihrer stickstoff haltigen Spaltprodukte noch nicht völlig erforscht; die Aufstellung einer empirischen Formel und die Ermittlung der Molekulargröße beruhten also in jener Zeit hauptsächlich auf der Analyse. Hierher gehören z. B. die ersten Arbeiten Schmiedebergs.

In den zweiten Abschnitt hinein fällt die Erforschung der stickstoffhaltigen Bestandteile an sich sowohl als auch hinsichtlich ihres Vorkommens in der echten Nucleinsäure. Es sind dies die Arbeiten von Kossel und Steudel, welch letzterer das molekulare Vorkommen von Guanin, Adenin, Cytosin und Thymin nachgewiesen hat. Bei der Aufstellung einer Formel ging $H$. Steudel von den Ergebnissen der vollkommenen Spaltung aus und suchte diese mit den Werten der Analyse in Einklang zu bringen.

Zur dritten Epoche gehören die Arbeiten von Levene und seinen Mitarbeitern, die durch partielle Hydrolyse einige höher molekulare Spaltprodukte darstellten und sie zur Aufstellung einer Konstitutionsformel benutzten. 
Die beiden letzteren Forscher nehmen gemeinschaftlich in der Nucleinsäure 4 Atome Pbosphor, vier Moleküle einer Hexose, sowie die bekannten vier Basen an; eine Meinungsverschiedenheit besteht hinsichtlich der Natur der Phosphorsäure, als welche der Phosphor offenbar vorkommt. Diese zuerst von Steudel ausgesprochene eVierzahl, der Hauptbestandteile der Nucleinsäure hat wohl allgemeine Anerkennung gefunden, und es mußte überraschen, daß vor einiger Zeit von Hildegarde Germann ${ }^{1}$ ) der Verdacht ausgesprochen wurde, die Thymonnucleinsäure enthielte nicht vier Moleküle Phosphorsäure, sondern die Zahl der vorhandenen Phosphorsäuremoleküle sei durch drei teilbar.

H. Germann geht von Versuchen von Levene und $\mathrm{Jacobs}^{2}$ ) aus, die ergeben hatten, daß in der. Hefenucleinsäure die Purinnucleotide durch saure Hydrolyse leichter zerstörbar sind als die Pyrimidinnucleotide. Diese für die Hefenucleinsäure geltenden Verhältnisse überträgt Verfasserin auf die echte Nucleinsäure, verfolgt quantitativ die Phosphorsäureabspaltung und kommt zu dem Schlusse, daß in der Thymonnucleinsäure ein Drittel der Phosphorsäure leicht abspaltbar sei, und mithin der Verdacht bestünde, daß die Anzahl der Phosphorsäuremoleküle nicht vier ist, sondern eine durch drei teilbare Zahl sein müsse.

Hierzu ist zu bemerken, daß dieser Versuch nur dann einigermaßen beweisend wäre, wenn bei der sauren Hydrolyse der Vorgang im Sinne von H. Germann durchaus quantitativ verlaufen würde, wenn also ein Drittel der Phosphorsäure ganz besonders leicht abspaltbar wäre, der Rest aber unter denselben Bedingungen fest gebunden bliebe, sodaß die Hydrolyse zeitlich eine breite Zone durchlaufen würde, in der überhaupt keine Phosphorsäureabspaltung stattfände.

Aber nicht einmal bei der Hefenucleinsäure verläuft dieser Vorgang so quantitativ; Levene und Jacobs sagen selbst: ${ }^{3}$ )

«Während die Purinkomplexe durch 2 stündiges Erhitzen mit 2\% iger Schwefelsäure vollkommen in Phosphorsäure,

1) Hil degarde Germann, Chem. Zentralbl. 1916, Bd. 2, S. 1036.

2) Levene und Jacobs, Chem. Ber., Bd. 44, S. 1027-32.

s) Ebenda. 
Ribose und Base gespalten werden, bleiben die Pyrimidinkomplexe bei dieser Behandlung größtenteils intakt.»

Unter diesen Bedingungen noch einen Analogieschlu $B$ auf die echte Nucleinsäure zu machen, scheint mir nicht angängig zu sein. Außerdem würde die Ansicht, daß nicht vier, sondern eine durch drei teilbare Anzahl P-Atome in der echten Nucleinsäure vorhanden wären, den bisherigen Anschauungen vollkommen widersprechen; sie würde auch widersprechen den Ergebnissen der Analyse; denn bei sehr zahlreichen Präparaten, die im Laufe der Zeit im hiesigen Institute angefertigt wurden, wurde stets das Verhältnis $\mathrm{N}: \mathrm{P}$ der Theorie gemäß zu 1,70 gefunden, das besagt aber, daß auf die vorhandenen 15 Stickstoffatome vier Phosphoratome kommen. Die Zahl der Stickstoffatome endlich ist nach den Versuchen von H. Steudel mit Sicherheit bekannt und auch allgemein anerkannt, da sie durch die Anwesenheit der vier gut definierten stickstoffhaltigen Spaltprodukte bedingt ist.

Ich glaube also keinen Grund zu haben, von der bisher angenommenen Zahl der Phosphoratome abzuweichen.

Legt man einer Nucleinsäureformel nun die Vierzahl der Hauptbestandteile zugrunde, nimmt man ferner nach Levene an, daß die Nucleinsäure sich aus vier einzelnen Nucleotiden zusammensetzt, so müßte man zu der einfachsten empirischen Formel kommen, indem man vier Moleküle Orthophosphorsäure, vier Moleküle einer Hexose, sowie die Molekulargewichte der vier Basen addiert und von der Summe 11 Moleküle Wasser - entsprechend den 11 Bindungen - subtrahiert. So käme man $z u$ der empirischen Formel

$$
\mathrm{C}_{43} \mathrm{H}_{65} \mathrm{O}_{35} \mathrm{~N}_{15} \mathrm{P}_{4} \mathrm{Na}_{4} \text {. }
$$

Mol.-Gew. 1526. $\mathrm{C}=33,01 \% \quad \mathrm{~N}=13,78 \% \quad \mathrm{P}=8,14 \%$.

Obgleich diese Formel in der Tat den Ergebnissen der Hydrolyse gerecht wird und außerdem, besonders hinsichtlich der Art der Phosphorsäure, die weitaus einfachste wäre, so konnte sie doch deswegen nicht angenommen werden, weil die theoretisch berechneten Analysenwerte mit den gefundenen nicht übereinstimmen wollten; denn stets findet man Werte, die mehrere Prozente höher liegen als die angegebenen be- 
rechneten (z. B. findet man für $\mathrm{C}=37 \%$, für $\mathrm{N}=15 \%$, für $P=9 \%$ ).

Es war also erforderlich, das Molekulargewicht der $\mathrm{Nu}-$ cleinsäure in der entwickelten Formel kleiner anzunehmen. Da nun die Molekulargewichte der Basen feststehen, als Kohlenhydrat aber eine «Hexose> allgemein angenommen wurde, und damals für eine Ablehnung einer «Hexose noch keine zwingenden Gründe vorlagen, so wurde von $H$. Steudel die Verkleinerung der Nucleinsäureformel dadurch berücksichtigt, daß er eine Tetrametaphosphorsäure annahm, weil durch diese Annahme das Molekulargewicht der Nucleinsäure kleiner (1388) wird, und mithin die gefundenen Analysenwerte mit den berechneten Werten besser in Einklang zu bringen waren. Später hielt er eine Tetraphosphorsäure für wahrscheinlich und suchte die. Mißlichkeit der zu hohen Werte absolut getrockneter Präparate dadurch $\mathbf{z u}$ beheben, daß er lufttrocken analysierte und im Molekül der Nucleinsäure Konstitutionswasser annahm.

Levene ${ }^{1}$ ) gibt eine Konstitutionsformel für die Nucleinsäure an, die sich hinsichtlich ihres Molekulargewichtes nicht wesentlich von dem oben angegebenen einfachsten Schema unterscheidet. Sie unterscheidet sich aber wesentlich von anderen Formeln dadurch, daß sie die Nucleinsäure - infolge der eigenartigen Bindung der Nucleotide - als eine sechsbasische Säure erscheinen läßt.

$\mathrm{Zu}$ dieser Formel habe ich folgendes zu bemerken:

1. Es fehlt der Beweis durch die Analyse. In Wirklichkeit findet man unter Zugrundelegung der Leveneschen Formel viel zu hohe Werte für $\mathrm{C}, \mathrm{N}$ und $\mathrm{P}$, weil das Molekulargewicht dieser Formel sich nicht wesentlich von dem Molekulargewicht der erwähnten einfachsten Formel unterscheidet. Nach Levene müßte man für das Natriumsalz 13,4\% $\mathrm{N}$ erwarten; statt dessen findet man aber $15 \% \mathrm{~N}$ ! Diese Werte wurden nicht allein in eignen Analysen gefunden (vgl. unten), vielmehr besagen die Analysen anderer Forscher dasselbe. So fand H. Steudel ${ }^{2}$ )

1) Levene und Jacobs, Journ. of Biol. Chem., Bd. 12, S. 412 (1912).

2) H. Steudel, Diese Zeitschr., Bd. 49, S. 408. 
Über die «Kohlenhydratgruppe` in der echten Nucleinsäure. 245

für das Kupfersalz 14,37 und 14,47\% N; Schmiedeberg ${ }^{1}$ ) für das Kupfersalz 13,90, 14,67 und 14,45\% N (in verschiedenen Präparaten). Berechnet man diese Werte auf das Natriumsalz, so haben gefunden:

$$
\begin{aligned}
& \text { H. Steudel: } \quad 14,81 \quad 14,91 \% \mathrm{~N} \\
& \text { Schmiedeberg: } 14,33 \quad 15,12 \quad 14,90 \% \mathrm{~N} \text {. }
\end{aligned}
$$

Vergleicht man ferner damit einen bereits von mir publizierten Stickstoffwert $(15,2 \%),{ }^{2}$ ) so muß man zu dem Schlusse kommen, daß in der Tat von den verschiedensten Autoren Werte gefunden werden, die um 15\% herumliegen. Dies ist aber um so bedeutungsvoller, als alle drei Autoren ihre Nucleinsäure nach verschiedenen Methoden herstellten. Schmiedeberg nach dem Kupfer-Alkaliverfahren, Steudel nach Neumann, und ich nach einem abgeänderten Neumannschen Verfahren. ${ }^{3}$ )

Man muß also zu dem Schlusse kommen, daß die Formel nach Levene nicht mit den Ergebnissen der Analyse in Übereinstimmung $\mathrm{zu}$ bringen ist.

2. Nach Angaben aller bisherigen Forscher ist die Nucleinsäure keine sechs-, sondern eine vierbasische Säure; es müßte also erst der Beweis erbracht werden, daß sie sechsbasisch ist. Für die vierbasische Natur der Nucleinsäure spricht die Zusammensetzung früher von mir dargestellter Farbsalze der Nucleinsäure. $\left.{ }^{4}\right)$ Da die benutzten Farbbasen ein hohes Molekulargewicht hatten und sich leicht mittels Alkali abscheiden und bestimmen ließen, so sind sie besonders geeignet zur Untersuchung der Basizität der Nucleinsäure, zumal sich die Farbnucleinate selbst wegen ihrer Wasserunlöslichkeit leicht durch doppelte Umsetzung darstellen ließen. Auch die Analyse derartiger Farbnucleinate ergab einen vierbasischen Charakter der Nucleinsäure.

Auf Grund dieser Überlegungen gewann ich den Eindruck, daß die wahre Höhe des Molekulargewichtes der Nucleinsäure

1) Schmiedeberg, Arch. für exper. Pathol., Bd. 43, S. 62 (1899).

2) R. Feulgen, Diese Zeitschr., Bd. 92, S. 158 (1914).

3) R. Feuilgen, Diese Zeitschr., Bd. 90, S. 261 (1914).

4) R. Feulgen, Diese Zeitschr., Bd. 84, S. 326. 
noch nicht richtig zum Ausdruck gebracht worden ist, daß dieses vielmehr von den bisherigen Autoren stets zu boch angenommen worden ist. Da nun die Anzahl der $\mathrm{C}_{-}, \mathrm{N}$ - und P-Atome ein für allemal feststeht, so bleibt nur die Annahme übrig, daB die Anzahl der 0- und H-Atome in den Formeln nicht der Wirklichkeit entspricht, da $B$ also die Nucleinsäure weniger 0 und H-Atome enthält, als in den landläufigen Formeln zum Ausdruck kommt. Da weiter eine Verkleinerung der Phosphorsäure zu hypothetisch wäre, so bleibt keine andere Möglichkeit als der Verdacht, daß in der echten Nucleinsäure nicht, wie bisher angenommen, ein Zucker von der Molekulargröbe $\mathrm{C}_{6} \mathrm{H}_{12} \mathrm{O}_{6}$ vorkommt, sondern ein bisher unbekanntes Kohlenhydrat mit zwar sechs Kohlenstoffatomen, aber mit weniger 0 - und H-Atomen als in einer Hexose vorkommen. In der Vermutung, daß es sich nicht um einen «Zucker " handelt, wurde ich durch die überaus leichte Verharzbarkeit des Kohlenhydrates bestärkt, welchem Umstande man es auch zuschreiben muß, daß es noch nicht gelungen ist, diesen Körper zu isolieren, obgleich er die Hälfte des Gewichtes der Nucleinsäure ausmacht.

Um der Kohlenhydratgruppe näher zu kommen, nahm ich das Studium der Thyminsäure auf, da ja in ihr die reduzierenden Gruppen von zwei Kohlenhydraten durch die Abspaltung des Guanins und Adenins freigeworden und dadurch zahlrëichen Eingriffen zugänglich gemacht waren. Es gelang, die Reduktionskraft der sehr zersetzlichen Thyminsäure zu erhalten und einen Körper zu gewinnen, der hinsichtlich der Verhältnisse $\mathrm{N}: \mathrm{P}$ und $\mathrm{C}: \mathrm{P}$ allen Anforderungen entsprach; aber die Analysenwerte absolut getrockneter Präparate waren auch hier mehrere Prozent zu hoch und bestärkten mich in der Annahme, daß das Kohlenhydrat in der Tat ein kleineres Molekulargewicht haben müsse, als einer Hexose $\mathrm{C}_{6} \mathrm{H}_{12} \mathrm{O}_{6}$ zukommt. Je mehr sich dieser Verdacht befestigte, desto verwickelter und übersichtlicher wurden die Verhältnisse; konnte doch unter den bisher bekannten « Kohlenhydraten * keines gefunden werden, auf das die Eigenschaften des Kohlenhydrates in der Nucleinsäure auch nur einigermaßen paßten. 
Über die ‘Kohlenhydratgruppe» in der echten Nucleinsäure. 247

Da wurde von E. Fischer ${ }^{1}$ ) eine neue Gruppe von Körpern entdeckt, die berufen sein sollte, Licht in die überaus schwierigen Verhältnisse der Nucleinsäure zu bringen, nämlich die Gruppe des Glucals. Wie ich in einer früheren kurzen Mitteilung dargelegt habe, ${ }^{2}$ ) bin ich zu der Überzeugung gekommen, daß in der echten Nucleinsäure in der Tat ein Vertreter jener Körperklasse vorkommt und keine «Hexose».

Diese neuen Körper sind dadurch gekennzeichnet, daß sie im Gegensatz zu den Hexosen

1. eine echte Aldehydgruppe enthalten, mithin fuchsinschweflige Säure färben und mit Phenylhydrazin Verbindungen vom Charakter der Hydrazone eingehen,

2. einen Furanring besitzen und deswegen eine grüne Fichtenspanreaktion geben,

3. sowohl mit Laugen als auch mit Säuren äußerst leicht verharzen,

4. eine Molekulargröße von $\mathrm{C}_{6} \mathrm{H}_{10} \mathrm{O}_{4}$ haben.

All diese Dinge, die einzeln schon bei einem unmittelbaren Abkömmling einer Hexose eine auffallende Erscheinung bilden würden, sind in ihrer Gesamtheit so charakteristisch, daß an ihnen die Gruppe des Glucals mit Sicherheit erkannt werden kann.

Auch die echte Nucleinsäure gibt sämtliche erwähnten Reaktionen teils direkt, teils nach Abspaltung der Purine durch milde Hydrolyse. Wird diese Hydrolyse so geleitet, daß der Prozeß quantitativ verläuft, so entsteht die Thyminsäure, über die in einer späteren Abhandlung berichtet werden soll; zum Nachweis der erwähnten Reaktionen ist ihre Isolierung nicht erforderlich, sodaß die Versuche mit Leichtigkeit an der Nucleinsäure selbst angestellt werden können.

\section{Ausführung der Reaktionen.}

0,2 $\mathrm{g}$ nucleinsaures Natrium werden im Reagenzglase in $2 \mathrm{ccm}$ Wasser im Wasserbade gelöst, zu der heißen Lösung $1 \mathrm{ccm} 2 \mathrm{n}$-Schwefelsäure zugegeben, und das Glas unter öfterem Umschütteln 3 Minuten im siedenden Wasserbade belassen. In dieser Zeit löst sich die anfangs ausgefallene Nucleinsäure voll-

1) E. Fischer, Chem. Ber., Bd. 47, S. 196 (1914).

2) R. Feulgen, Diese Zeitschr., Bd. 92, S. 157 (1914). 
ständig auf. Dann kühlt man unter der Wasserleitung ab und neutralisiert genau mit 2 n-Natronlauge, wobei eine Trübung von ausfallendem Guanin aufzutreten pflegt.

\section{Aldehydreaktion.}

Zu einigen Kubikzentimetern fuchsinschwefliger Säure ${ }^{1}$ ) gibt man einen Tropfen der Hydrolysenflüssigkeit: Intensive Violetlfärbung nach wenigen Augenblicken. Die Reaktion ist äußerst empfindlich; ein einziger 'Tropfen einer $1 \%$ igen Lösung von reinem thyminsauren Baryum gibt die Reaktion noch deutlich. Versetzt man einige Kubikzentimeter ammoniakalischer Silberlösung mit einigen Tropfen der Flüssigkeit, so tritt nach einiger Zeit Reduktion ein. Es bildet sich aber kein oder nur ein sehr unvollkommener Silberspiegel, sondern das reduzierte Silber bleibt kolloidal in Lösung, daher intensive Braunfärbung. Ein weiterer Beweis für die Aldehydnatur der freigewordenen reduzierenden Gruppen bildet die Eigentümlichkeit des thyminsauren Baryums, mit Phenylhydrazin Verbindungen einzugehen, in denen auf je ein Kohlenhydrat ein Molekül Phenylhydrazin kommt, und die infolgedessen den Charakter der Hydrazone, nicht der Osazone tragen. Auch über diesen Körper werde ich in Kürze Bericht erstatten.

\section{Die Fichtenspanreaktion.}

Ein Fichtenspan wird unter Erwärmen gut mit der Hydrolysenflüssigkeit getränkt und sodann in einem Reagenzglase Salzsäuredämpfen ausgesetzt, die durch Erhitzen von etwas konzentrierter Salzsäure in der Kuppe des Reagenzglases erzeugt werden: Der Stab nimmt eine schöne tiefgrüne Farbe an.

\section{Leichte Verharzbarkeit.}

Daß der Körper mit Säuren überaus leicht verharzt, geht aus der massenhaften Huminbildung bei starker Säurehydrolyse hervor, sowie aus dem Umstande, daß es noch nicht gelungen ist, das Kohlenhydrat durch Hydrolyse darzustellen. Die leichte

1) Eine 0,5\% ige Lösung von Fuchsin wird mit $\mathrm{SO}_{2}$ entfärbt, und das überschüssige $\mathrm{SO}_{2}$ im Vakuum entfernt. 
Ther die sKohlenhydratgruppe, in dor echten Nucleinshure. 249

Verharzbarkeit mit Laugen läßt sich leicht zeigen, wonn man dio Hỵdrolysenflüssigkeit mit etwa 1s Volumen $33 \%$ iger Natronlauge versetzt: schon in der Kälte färbt sich die Masse bald rotbraun: beim Erhitzen tritt die Fürbung augenblicklich untor Entwicklung eines charakteristischen aromatischon (ieruchos ein.

\section{Molekulargowicht.}

Das Molekulargewicht des Kohlenhydrats selbst kann natürlich erst nach Isolierung desselben bestimmt werden. Da es aber - bei bekinnten sonstigen Bestandteilen bestimmenden Einfluß au das Molekulargewicht der Nucleinsäure ausübt, so fällt die Frage des Mtolekulargewichtes des Kohlenhydrates mit dem Problem des Molekularyewichtes der Nucleinsäure zusanmen. Der Beweis für das Molekulargewicht des Kohlenhydrats besteht also nicht nur in der Analogie mil der Molekularyörbe des Crluculs und seinor Verwandten, sondern die Beweisketle für meine Anschaunngen wird geschlossen durch die Analysen des nucleinsauren Natriums sowie auch möglichst vielor direkten Derivale der Nucleinsäure, in denen die Kohlenhydratkomplexe noch unverändert, oder doch nach bekanuten Gesetzen veründert vorkommen. Es mußte also mein Bestreben sein, solche Derivate in größerer Zilhl darzustellen, damit ihre Analysen als Stütze für meine Ansicht über das Molekulargewicht dor Nucleinsüure, und damit auch des Kohlcuhydrates, betrachtot werden können. Je mannigfaltiger die durgestellten Derivate, desto größere Beweiskraft mußte ihnen zukommen.

In Frage kommen zunächst die Analysen:

1. des $\alpha$-nucleinsauren Nilriums,

2. des $\beta$-nucleinsauren Natriums,

3. des thyminsauren Baryums,

4. mehrere Verbindungen des thyminsauren Baryums, entstanden auf Grund der freien Aldehydgruppe in diesem Körper, (z. B. hydrazonähnliche Substanzen), Verbindungren, die zugleich weitere Beweise für die Aldehydnalur des Kohlenhydrates sind. 
Die Analysen der unter 2 bis 4 genannten Stoffe werde ich später bei Gelegenheit der Besprechung dieser Körper bringen; jetzt soll nur das Molekulargewicht des $\alpha$-nucleinsauren Natriums abgehandelt werden.

Das zu analysierende nucleinsaure Natrium.

Am empfehlenswertesten zur Analyse ist das nucleinsaure Natrium, weil es das direkte Endprodukt der Neumannschen Darstellungsweise bildet. Es hat vor der freien Nucleinsäure den Vorzug unbegrenzter Haltbarkeit. Kupfersalze sind schwerer zu verarbeiten und auch schwer natriumfrei zu bekommen. Ein Blick auf die oben angegebenen und auf die Natriumsalze umgerechneten Analysenzahlen ganz unparteiischer Autoren zeigt, daß auch diese Werte mit meinen Anschauungen über die Molekulargröße der Nucleinsäure vorzüglich übereinstimmen, und daß Präparate, auch nach verschiedenen Methoden dargestellt, dennoch zu gebrauchen sind. Besonders vorsichtig jedoch muß man bei der Darstellung des nucleinsauren Natriums zu Werke gehen, wenn man etwa über die Thyminsäure andere Verbindungen herstellen will; denn es ist $\mathrm{zu}$ beachten, daß die hochmolekularen Derivate noch nicht zur Krystallisation gebracht werden konnten, daß vielmehr meistens die Alkoholfällung $\mathrm{zu}$ ihrer Isolierung benutzt werden muß, eine Isolierungsmethode, der keine auswählende Wirkung hinsichtlich zersetzter und unzersetzter Nucleinsäurederivate zukommt. Abgesehen davon, daß also nur solche Derivate in Frage kommen, die durch quantitativen Verlauf der Reaktion entstehen, muß vor allen Dingen das Ausgangsmaterial, nämlich das nucleinsaure Natrium von durchaus einwandfreier Beschaffenheit sein.

Ich muß an dieser Stelle an die große Gefahr erinnern, der die Präparate bei zu langer Einwirkung der Natronlauge bei der Neumannschen Verflüssigung der Thymusdrüsen ausgesetzt sind; anderseits soll aber alles Eiweiß aus dem Proteid abgespalten werden. Nun ist aber zu bedenken, daß die Biuretprobe sehr empfindlich ist und selbst dann noch positiv ausfällt, wenn durch die Analyse ein zu hoher Stickstoffgehalt nicht mehr nachweisbar ist, ferner, daß eine zu lange Ein- 
Über die ‘Kohlenhydratgruppes in der echten Nucleinsăure. 251

wirkung der Natronlauge unter allen Umständen das Präparat verdirbt (es entsteht dann keine $\beta$-Nucleinsäure, sondern ein Zersetzungsprodukt) ${ }^{1}$ ); man wird mithin lieber eine schwache Biuretprobe in den Kauf nehmen, als die Natronlaugewirkung zu lange ausdehnen, zumal infolge der hochkolloidalen Eigenschaften des wirklich intakten $\alpha$-nucleinsauren Natriums (mit einem Gallertschmelzpunkt von über $50^{\circ}$ ) ein Teil der biuretgebenden Stoffe durch die Alkoholfällung des Präparates leicht adsorbiert wird und im Niederschlage erscheint.

Früher ${ }^{2}$ ) wurde gezeigt, daß das übliche halbstündige Erhitzen der Thymusdrüsen mit $2 \%$ iger Natronlauge allein nicht imstande ist, ein einigermaßen eiweißfreies Präparat $z u$ gewährleisten, daß vielmehr ein nachträgliches mehrstündiges Erhitzen des Rohproduktes mit Natriumacetatlösung zweckmäßig angewendet wird. Die nach dieser Darstellungsmethode erzielten Präparate haben sich als sehr geeignet sowohl zur Analyse als auch zur Darstellung der Derivate der Nucleinsäure erwiesen. Auf einige Dinge, die, wie sich bei längerer Anwendung der Methode herausgestellt hat, eine glatte Darstellung wesentlich erleichtern, soll am Schlusse dieser Abhandlung in Form eines Anhanges eingegangen werden.

Das lufttrockene nucleinsaure Natrium enthält je nach dem Feuchtigkeitsgehalte des Raumes, in dem es gelegen hat, noch etwa 10-30\% (meist etwa 15\%) Wasser, dessen größter Teil schon bei gewöhnlicher Temperatur über Schwefelsäure im Vakuum weggeht. Zur Entfernung der letzten Reste ist eine Temperatursteigerung notwendig. Die zulässige Temperatur ergab sich aus meinen früheren Versuchen, ${ }^{3}$ ) wobei festgestellt wurde, daß das nucleinsaure Natrium, trocken erhitzt, allmählich von der a-Form in die b-Form übergeht, was am Sinken des Gallertschmelzpunktes kontrolliert wurde. Es zeigte sich, daß bei $80^{\circ}$ innerhalb mehrerer Stunden noch keine Abnahme des Gallertschmelzpunktes zu beobachten war; dementsprechend trockne ich die Präparate stets im Vakuum über

) R. Feulgen, Diese Zeitschr., Bd. 91, S. 165 (1914).

2) R. Feulgen, Diese Zeitschr., Bd. 90, S. 263 (1914).

3) R. Feulgen, Diese Zeitschr., Bd.91, S. 170 (1914). 
Phosphorpentoxyd bei $80^{\circ} 4$ Stunden lang. Da über die Natur der b-Form so gut wie nichts bekannt ist, so soll sie hier zunächst ausgeschlossen und Gegenstand einer besonderen Besprechung werden.

Unter den genannten Bedingungen ist das Wasser praktisch verschwunden; zwar nimmt mit der Erhöhung der Temperatur das Gewicht der Substanz noch kontinuierlich $a b$, doch ist diese Abnahme bis zur Zersetzung nicht erheblich und macht im Stickstoffgehalte etwa $0,1 \%$ aus. Ich analysiere meist lufttrocknes Material und nehme in einer besonderen Probe eine Wasserbestimmung vor.

\section{Zur Elementaranalyse von Nucleinsäuren.}

Die Elementaranalyse von Nucleinsäuren ist dadurch kompliziert, daß wegen des hohen Phosphorgehaltes leicht von Asche eingeschlossene phosphorhaltige Kohle zurückbleibt, die schlecht oder garnicht verbrennbar ist. Im allgemeinen kann man bei der Verbrennung von Nucleinsäuren drei Fälle unterscheiden:

1. Es liegt freie Nucleinsäure oder ein Salz mit einer organischen Base vor. In diesem Falle erscheint im Rückstande freie Phosphorsäure bezw. Metaphosphorsäure. Verbrennt man freie Nucleinsäure nach Dennstedt, so bleibt stets eine erhebliche Menge unverbrennbarer Kohle zurück. Dennsted ${ }^{1}$ ) empfiehlt, zur Verbrennung phosphorhaltiger Eiweißverbindungen ein poröses Tonschiffchen zu verwenden. Die bei der Verbrennung gebildete Phosphorsäure soll dann von dem porösen Ton des Schiffchens aufgesaugt werden, wodurch die nunmehr von der Phospborsäure befreite Kohle leicht und vollständig verbrennbar wird. Bei der Nucleinsäure versagt aber diese Methode. Es wird keine Phosphorsäure von dem porösen Tonschiffchen aufgenommen, und selbst eine eingeschaltete Extraktion des Schiffcheninhaltes führt nicht zum Ziele, immer bleibt eine vollkommen unverbrennliche graphitähnliche Kohle zurück.

2. Es liegt nucleinsaures Natrium vor, bei dem auf je ein Atom Phosphor ein Atom Natrium kommt. Als Rückstand

') Diese Zeitschr., Bd. 52, S. 181 (1907). 
Über die ‘Kohlenhydratgruppe» in der ecbten Nucleinsäure. 253

bildet sich in diesem Falle schmelzbares Natriummetaphosphat. Zwar wird auch dieses nicht von dem porösen Tonschiffchen aufgenommen, aber bei langer Fortsetzung der Verbrennung gelingt es doch meist, alle Kohle zur Verbrennung zu bringen, da bei Anwendung hoher Temperaturen die dickflüssige geschmolzene Asche sich fortwährend in langsamer Bewegung befindet, und allmählich alle Kohle mit dem Sauerstoff in Berührung kommt. Solche Fälle sind schon bei Beginn der Verbrennung daran zu erkennen, daß die Substanz bei beginnender Verkohlung anfängt, sich stark zu blähen.

3. Es liegt ein nucleinsaures Natrium vom Typus des guanylsauren Natriums vor. Hier kommen auf je ein Atom Phosphor zwei Atome Natrium; es bildet sich also Natriumpyrophosphat und ein solcher Rückstand schmilzt nicht bei der angewandten Temperatur. Derartige Präparate blähen sich bei der Verbrennung nicht, sondern verkohlen ohne merkliche Bewegung. Auch hier bleibt Kohle in der Asche eingeschlossen, diese läßt sich aber leicht extrahieren und die von der Asche befreite Kohle verbrennt glatt. Zur Extraktion der großen Aschenmengen hat sich folgendes Verfahren bewährt:

Ein mit einem Filtrierpapier beschickter Büchnerscher Trichter wird zur Hälfte mit einem wässerigen Brei von geglühtem Seesande angefüllt und in diesen Brei das poröse Tonschiffchen so eingesenkt, daß nur ein kleiner Rand übersteht. Wird jetzt evakuiert, so wird Flüssigkeit sehr leicht nicht nur durch den Sand, sondern auch durch das Schiffchen hindurchgesaugt und es gelingt sehr rasch, den Inbalt des Schiffchens und dieses selbst durch Hineinbringen erst von verdünnter Salzsäure, dann von Wasser vollständig von Asche zu befreien. Nach dem Trocknen wird die Verbrennung fortgesetzt.

Auch Baryumsalze von hochmolekularen Nucleinsäurederivaten (thyminsaures Baryum) sind sehr schwer zu verbrennen. Hierbei ist es nicht möglich, die Asche vollständig von der Kohle zu befreien.

Alle die genannten Unbequemlichkeiten lassen sich vermeiden, wenn man - wie es bis jetzt auch wohl meist geschehen ist - die Substanz vor der Verbrennung mit geeigneten Stoffen

Hoppe-Seyler's Zeitschrift f. physiol. Chemie. C. 
vermischt. Als einfachstes hat sich mir Kupferoxyd bewährt, das aber mit der Substanz sehr innig vermischt werden muB, sollen nicht schwerverbrennliche Knötchen entstehen. Da aber in diesem Falle das Verfahren nach Dennstedt bei der Elementaranalyse von Nucleinsäuren keine entscheidenden Vorzüge mebr hat, so wende ich die alte Liebigsche Nethode an, bis es gelungen ist, der erwähnten Schwierigkeiten in einfacher Weise Herr zu werden.

Das Molekulargewicht der Nucleinsäure ergibt sich nach den bisherigen Ausführungen einfach durch Addition von vier Molekülen Orthophosphorsäure, vier Molekülen des Kohlenhydrats von der Molekulargröße $\mathrm{C}_{6} \mathrm{H}_{10} \mathrm{O}_{4}$ und je einem Molekül Guanin, Adenin, Gytosin und Thymin und Abzug von 11 Molekülen Wasser, entsprechend den 11 Bindungen. Daraus ergibt sich für das Natriumsalz die empirische Formel $\mathrm{C}_{43} \mathrm{H}_{47} \mathrm{O}_{25} \mathrm{~N}_{15} \mathrm{P}_{4} \mathrm{Na}_{4}$ und das Molekulargewicht 1390, während unter Zugrundelegung einer Hexose $\mathrm{C}_{6} \mathrm{H}_{12} \mathrm{O}_{6}$ ein Molekulargewicht von 1526 gefordert werden müßte. Die neue Formel ist also um acht Sauerstoff- und acht Wasserstoffatome oder um $136 \mathrm{~d}$. h. um fast $9 \%$ kleiner. Der Unterschied macht im Stickstoffgehalte fast $2 \%$,im Kohlenstoffgehalte $5 \%$ aus; es sind dies die eingangs erwähnten bisher unerklärlichen und von Lev en e nicht berücksichtigten Unterschiede. Abgesehen von den oben erwähnten Analysenzahlen älterer Untersuchungen von anderen Autoren sprechen meine neueren Werte für diese Ansicht.

\section{Präparat $\mathrm{I}$.}

$0,2322 \mathrm{~g}$ verloren bei $78^{\circ}$ über $\mathrm{P}_{2} \mathrm{O}_{5}=0,0329 \mathrm{~g}$ Wasser, 0,2604 , entsprachen $24,4 \mathrm{ccm}$ n/10-Säure (Kjeldahl), 0,2583 , > 24,3 , > 0,1613 , > 22,5 , $n_{i 2}$-Lauge (Neumann), 0,1043 , > 15,0 , > 0,3733 , gaben $0,4323 \mathrm{~g} \mathrm{CO}_{2}$ und $0,1591 \mathrm{~g} \mathrm{H}_{8} 0$.

Berechnet auf trockne Substanz.

\begin{tabular}{|c|c|}
\hline $\begin{array}{c}\text { Berechnet für } \mathrm{C}_{43} \mathrm{H}_{47} \mathrm{O}_{25} \mathrm{~N}_{15} \mathrm{P}_{4} \mathrm{Na}_{4} \text { : } \\
\text { C } \quad 37,12 \\
\text { H } \quad 3,49\end{array}$ & $\begin{array}{l}\text { Gefunden: } \\
36,8 \\
3,79\end{array}$ \\
\hline N 15,12 & $15,30 \quad 15,36$ \\
\hline P $\quad 8,93$ & 9,01 \\
\hline
\end{tabular}




\section{Präparat II.}

$0,3029 \mathrm{~g}$ verloren bei $78^{\circ}$ über $\mathrm{P}_{8} \mathrm{O}_{6}=0,0476 \mathrm{~g}$ Wasser, 0,2232 > entsprachen 20,6 ccm n/10-Säure (Kjeldahl), 0,2453 ,

0,1142 ,

22,6 ,

0,1202 ,

$15,4, n / 2$-Lauge (Neumann),

16,6

Berechnet auf trockene Substanz.

$$
\begin{array}{cccc}
\text { Berechnet für } & \mathrm{C}_{45} \mathrm{H}_{47} \mathrm{O}_{25} \mathrm{~N}_{15} \mathrm{P}_{4} \mathrm{Na}_{4}: & \multicolumn{2}{c}{\text { Gefunden: }} \\
N & 15,12 & 15,33 & 15,32 \\
\text { P } & 8,94 & 8,89 & 9,09 .
\end{array}
$$

Nun ist es Levene gelungen, ${ }^{1}$ ) zwei partielle Spaltprodukte aus der Nucleinsäure darzustellen, die deswegen besonders wertvoll sind, weil sie in Form krystallisierender Salze erhalten wurden. Diese Spaltprodukte ähneln in ihrer Zusammensetzung sehr den einfachen Nucleotiden, aus denen Levene sich die Nucleinsäure aufgebaut denkt, da sie sowohl Phosphorsäure als auch Kohlenhydrat, sowie Base enthalten. Die von Levene veröffentlichten Analysenzahlen sind unter Zugrundelegung einer Hexose $\mathrm{C}_{6} \mathrm{H}_{18} \mathrm{O}_{6}$ für das Kohlenhydrat berechnet, und man sollte meinen, daß sie vor allem dazu berufen wären, die Frage der Molekulargröße des Kohlenhydrats durch Analyse zu lösen.

Dies ist aber nicht der Fall. Die genannten Körper enthalten nämlich auf ein Molekül des Kohlenhydrates nicht ein, sondern $\mathrm{z}$ w ei Moleküle Phosphorsäure und sind obendrein vierbasisch, sodaß das Molekül der untersuchten Brucin- oder Baryumsalze im Verhältnis zu dem Kohlenhydrat viel größer ist, als das bei der Nucleinsäure selbst der Fall ist, bei der auf ein Molekül Kohlenhydrat nur ein Molekül Phosphorsäure und nur ein Natriumatom von kleinem Atomgewichte kommen. Deswegen sind auch die Unterschiede in den Analysenzahlen, das eine Mal mit einer Hexose, das andere Mal mit einem Kohlenhydrat von der Molekulargröße $\mathrm{C}_{6} \mathrm{H}_{10} \mathrm{O}_{4}$ berechnet, nur gering, während bei der Nucleinsäure selbst, wie gesagt, diese Unterschiede mehrere Prozente ausmachen. Eine Zusammenstellung wird dies ersichtlich machen:

1) Levene u. Jacubs, Journ. of Biol. Chem., Bd. 12, S. 417. 
1. Ba-Salz der Hexo-Thymidin-Diphosphorsäure.

\begin{tabular}{c|c|c|c}
\hline & $\begin{array}{c}\text { Bcrechnet } \\
\text { nach Levene }\end{array}$ & $\begin{array}{c}\text { Berechnet } \\
\text { nach Feulgen }\end{array}$ & $\begin{array}{c}\text { Gefunden } \\
\text { von Levene }\end{array}$ \\
\hline C..... & 18,44 & 19,2 & 18,12 \\
H..... & 1,96 & 2,06 & 2,13 \\
N..... & 3,91 & 4,09 & $4,114,18$ \\
P.... & 8,66 & 9,06 & 9,06 \\
Ba..... & 38,30 & 40,1 & 38,54
\end{tabular}

II. Ba-Salz der Hexo-Cytidin-Diphosphorsäure.

\begin{tabular}{l|r|r|r}
\hline C..... & 17,08 & 17,9 & 17,06 \\
H. . . . & 1,85 & 1,95 & 2,49 \\
N..... & 5,96 & 6,27 & 5,71 \\
P..... & 8,82 & 9,27 & 9,11 \\
Ba. . . . & 39,00 & 41,0 & 39,55
\end{tabular}

Beim Vergleiche dieser Werte ist noch zu beachten, daß die Ba-Salze dieser Körper nicht krystallisiert erhalten wurden, und deswegen auch die von Levene gefundenen Werte manchmal erheblich von den von Levene selbst geforderten Zahlen abweichen.

Endlich muß ich an dieser Stelle einer Veröffentlichung Levenes aus dem Jahre 1912 gedenken. Danach hat Levene ${ }^{1}$ ) durch Fermentspaltung aus der echten Nucleinsäure ein Guanosinhexosid dargestellt, aus diesem durch halbstündiges Kochen mit 2\% iger Schwefelsäure die Hexose abgespalten und aus ihr ein Osazon dargestellt. Diese Versuche sind in Form einer sehr kurzen Mitteilung veröffentlicht. Sie wären für die Nucleinsäureforschung von del allergrößten Bedeutung und würden mit meiner Ansicht vollkommen in Widerspruch stehen. Leider hat Levene bis jetzt (in der allerletzten Zeit war mir die amerikanische Literatur allerdings nur lückenhaft zugänglich) noch keine näheren Angaben über die Darstellung, speziell über die Natur des benutzten Fermentes gemacht, sodaß ich

1) Levene u. Jacobs, Journ. of Biol. Chem., Bd. 12, S. 377 (1912). 
heute noch nicht - obgleich die Veröffentlichung bereits 5 Jahre zurückliegt - in der Lage war, zu diesen Versuchen Stellung zu nehmen.

Zum Schlusse sei mir eine Vermutung darüber gestattet, ob vielleicht das Glucal selbst in der Nucleinsäure vorkommt, oder nur ein ihm analog gebauter Vertreter der offenbar großen Klasse. Die erstere Meinung wäre ja immerhin wahrscheinlich, wenn man bedenkt, daß das Glucal ein Derivat des Traubenzuckers und auch aus diesem dargestellt worden ist. Ich glaube aber annehmen zu können, daß das in der Nucleinsäure vorkommende Kohlenhydrat nicht mit dem Glucal identisch ist. Denn erstens ist der Geruch, der bei der alkalischen Verharzung des Glucals auftritt, ein anderer als der, wie er bei der alkalischen Zerstörung des fraglichen Kohlenhydrates z. B. in dem oben beschriebenen Versuche sich bemerkbar macht; dann aber ist das Kohlenhydrat in der Nucleinsäure offenbar noch viel empfindlicher gegen Säuren und Alkalien, als es selbst das Glucal ist; denn bei derselben Einwirkung (milde Hydrolyse mit Barytwasser), mittels welcher E. Fischer das Glucal aus seiner Acetylverbindung abspaltet, tritt bei der Thyminsäure unfehlbar vollkommene Verharzung ein.

Im weiteren Verlaufe meiner Untersuchungen will ich versuchen, das Kohlenhydrat (ev. mit Hilfe der beiden freien Aldehydgruppen in der Thyminsäure) in ein stabileres Derivat umzuwandeln, das eine Abspaltung von der Phosphorsäure aushält; derartige Versuche behalte ich mir vor.

Die Untersuchungen wurden mit Mitteln aus der Gräfin BoseStiftung ausgeführt.

\section{Anhang.}

Notizen zur Darstellung der Nucleinsäure.

Nachdem die früher beschriebene Methode ') zur Darstellung von nucleinsaurem Natrium aus Kalbsthymus in sehr vielen Fällen angewendet worden ist, haben sich folgende kleine Abweichungen, die allerdings nicht das Wesen der Methode beeinflussen, sondern nur eine glatte Darstellung erleichtern, als empfehlenswert erwiesen.

1) R. Feulgen, Diese Zeitsıhr., Bd. 90, S. 261 (1914). 
258 R. Feulgen, Die ‘Kohlenhydratgruppes in der echten Nucleinsäure.

1. Zu Seite 267, Zeile 20:

Die Filtration wird nicht durch ein Koliertach, sondern darch ein Faltenfilter vorgenommen, das später zusammen mit dem Rückstande ausgekocht wird.

2. Zu Seite 268 oben.

Die erste Rohfällung muß mit Natriumacetatlösung längere Zeit erhitzt werden. Nun enthält die Masse aber noch von der Alkoholfällung her Reste Alkohol, die in zweierlei Weise die weitere Verarbeitang stören können: Einerseits neigt der verdampfende Alkohol sehr zu Siedeverzug, sodaß die Flüssigkeit beim Erhitzen trotz Siedesteinchen heftig stößt, anderseits können sich während der nachfolgenden Filtration in Filter Alkoholdampfblasen entbinden und dasselbe verstopfen, sodaB die Filtration sehr langsam verläuft. Der Alkohol wird zweckmäBig entfernt, indem man die Erhitzung nicht am Rückflubrobr, sondern in einem offenen Kolben vornimmt.

3. Zu Seite 268, Zeile 15.

Die zweite Fällung wird zweckmäBig mit siedendem Alkohol in der auf $80^{\circ}$ erwärmten Lösung vorgenommen. In diesem Falle fällt das nucleinsaure Natrium weich aus und klebt sofort am Boden fest. Durch Peitschen mit einem Holzstabe kann die Mutterlauge leicht von schwebenden Partikelchen bis zur Klärung befreit und dann einfach abgegossen werden. Man erspart so die Prozedur des Absaugens. Durch Schwenken des noch heißen Topfes wird der größte Teil des noch anhaftenden Alkohols zum Verdampfen gebracht. Durch Aufstellen auf das Wasserbad kann dieser Vorgang vollendet werden. Der Rückstand wird unter allmählicher Verwendung von 1 Liter heißen Wassers aus dem Kessel herausgelöst, in einen 2 Literkolben gebracht und nach S. 269, Zeile 2 weiterbehandelt.

4. Zu Seite 269, Zeile 6.

Die dritte Fällung wird zweckmäßig in der Kälte vorgenommen, damit man ein poröses Präparat erhält, das sich mit Alkohol leicht entwässern läßt. $\mathrm{Da}$ jedoch in der Kälte die Lösung eine feste Gallerte bildet, verfährt man derart, daß man zu der heißen mit etwas Natriumacetat versetzten Lösung vorsichtig unter Umrühren kalten Alkohol bis zur beginnenden Trübung zusetzt, dann abkühlen läßt und nun erst den Rest des Alkohols zur eigentlichen Fällung zufügt. 